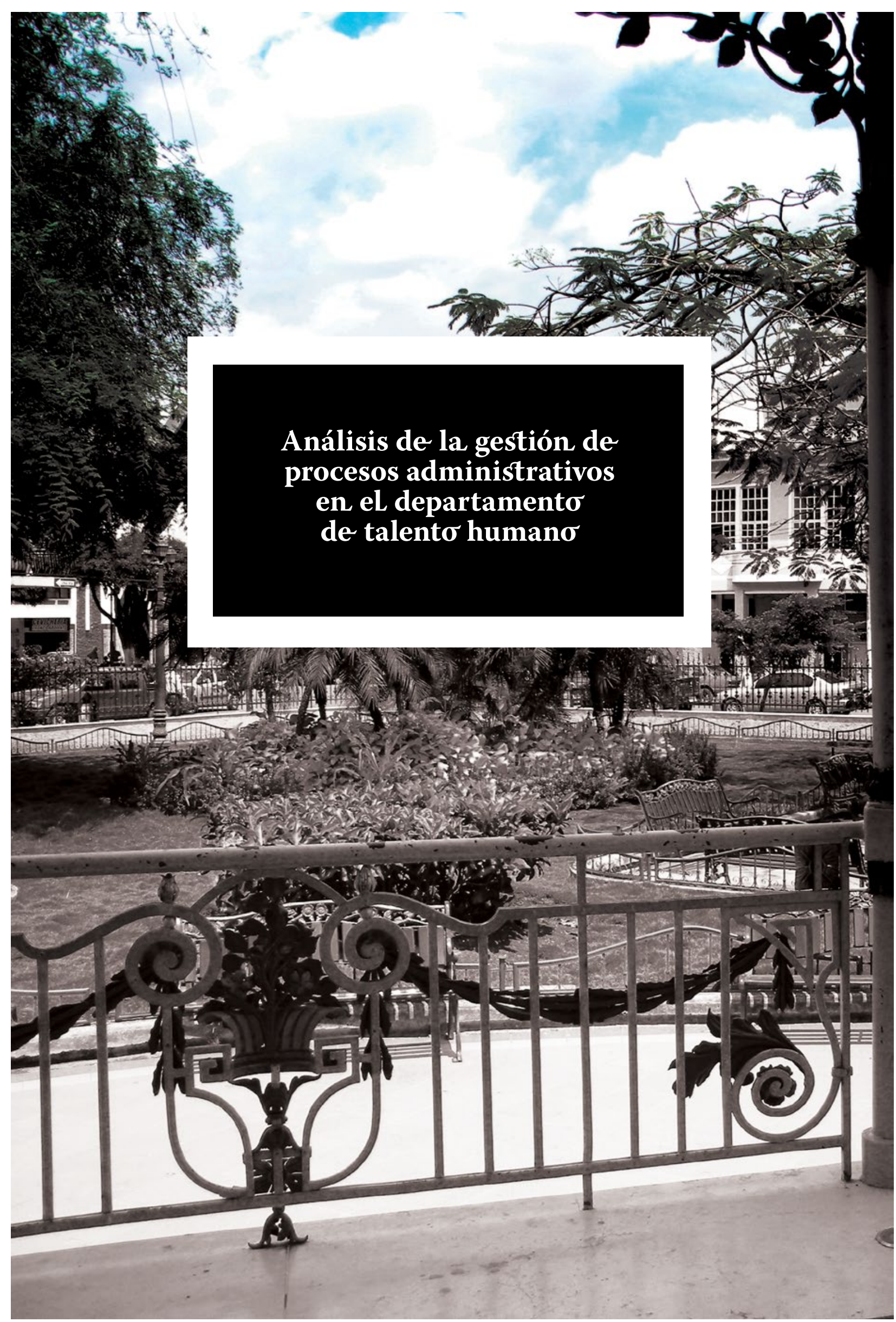




\section{ANÁlisis de LA Gestión de PROCESOS ADMINISTRATIVOS EN EL DEPARTAMENTO DE TALENTO HUMANO}

\section{ANAlysis Of THE MANAgement OF AdMinistrative PROCESSES IN THE DEPARTMENT OF HUMAN TALENT.}

\section{RESUMEN}

El presente trabajo plantea el análisis de la gestión de procesos administrativos en el departamento de talento humano en una empresa de transportación masiva, centrado en examinar la situación actual de la empresa, e identificar los factores internos y externos que afectan de alguna manera la gestión del departamento de talento humano. Se aplicó entrevista a jefes de áreas, y personal involucrado en la gestión del talento humano, se tomó una muestra conformada por el personal, administrativo y operativo, quienes proporciona ron la infor mación necesaria para la investigación. Con los resultados obtenidos, se detectaron falencias en los procesos de gestión administrativa, lo que les impide aportar de manera eficiente en la ejecución de tareas y procedimientos dentro de la empresa, así como también la falta de funciones bien estructuradas y definidas en la estructura de la empresa, lo que ocasiona pérdida de tiempo en las labores encomendadas, previo al reclutamiento y selección del puesto requerido. Finalmente, la empresa investigada, carece de procesos de gestión del talento humano que le permita administrarlos de manera eficiente y a la vez contribuir al cumplimiento de los objetivos estratégicos trazados.

PALABRAS CLAVES: Gestión de talento humano; habilidades; manual de funciones; procesos administrativos.

Copyright (c) Revista San Gregorio 2019. ISSN: 1390-7247; eISSN: 2528-7907 @

\section{ABSTRACT}

This paper presents the analysis of the management of administrative processes in the department of human talent in a mass transportation company, focused on examining the current situation of the company, and identify internal and external factors that affect in some way the management of the human talent department. An interview was applied to heads of areas, and personnel involved in the management of human talent, a sample was taken composed of the staff, administrative and operational, who provided the necessary information for the investigation. With the results obtained, shortcomings were detected in the administrative management processes, which prevents them from efficiently contributing to the execution of tasks and procedures within the company, as well as the lack of well-structured and defined functions in the structure of the company. The company, which causes loss of time in the tasks entrusted, prior to the recruitment and selection of the required position. Finally, the researched company lacks human talent management processes that allow it to manage them efficiently and at the same time contribute to the fulfillment of the strategic objectives set.

KEYWORDS: Administrative processes; management of human talent; manual of functions; skills.

\section{Mariana Bustamante Chong}

1.

$\sim$

Universidad Estatal del Sur de Manabí. Manabí. Ecuador.

mariana.bustamante@unesum.edu.ec

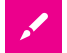

1.

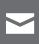

\section{Vanessa CaAmaño Bustamante}

Estudiante Universidad San Gregorio de Portoviejo. Manabi. Ecuador. e.vecaamano@sangregorio.edu.ec
César Bustamante Chong

1.

Universidad Tecnológica ECOTEC. Guayaquil. Ecuador

$\sim$

cbustamante@ecotec.edu.ec

\section{Franklin Cabezas Galarza}

Universidad de Guayaquil. Guayaquil. Ecuador.

franklin.cabezasg@ug.edu.ec 


\section{INTRODUCCIÓN}

Después de diferentes consensos basados en identificar la división expuesta por Henry Fayol en la administración con los elementos: previsión, organización, dirección y coordinación; en el que establece que en todas las empresas industriales, independientemente de su complejidad, y magnitud, hay seis grupos de funciones identificadas como: funciones técnicas, funciones comerciales, funciones financieras, funciones de seguridad, funciones contables y funciones administrativas (Fayol, 1982) se considera que este autor fue el primero en proponer un modelo de procesos administrativos y define la acción de administrar como proveer, organizar, dirigir, coordinar y controlar.

Según (Gulick, 1992) citado por (Chiavenato, 2009) "propone siete elementos de la administración como las principales funciones del administrador: a) planeamiento (planning); b) organización (organizing); c) asesoría (sic) aquí, se debe considerar que Chiavenato comete un error importante al traducir del inglés staffing por "asesoría”. La traducción correcta sería "integración" (de personal) o algún otro término equivalente.

Según (Baguer, 2001) en la mayoría de los casos, las empresas concentran su esfuerzo con el fin de alcanzar sus objetivos a corto plazo de la manera más eficaz, (no siempre es la mejor opción) y destinan sus recursos importantes y principales en la resolución de los problemas del momento.

En la mayoría de las empresas las personas se comportan de manera distinta con las que son, es decir, que el comportamiento de una persona va en respuesta a cómo los otros se comportan con ella y depende en gran medida por la motivación del trabajador para desempeñarse.
Por su parte (Rodríguez, 2007) añade que los recursos humanos los forman las personas que trabajan para una institución, quienes poseen habilidades y conocimientos acerca del sistema de trabajo, por lo que son de gran valor para los administradores.

Concluye (Quintanilla, 2002) que la gestión del talento humano está orientada a la conformación y optimación del comportamiento de las personas, desde las expectativas de las organizaciones, pero además hay que tomar en cuenta las aspiraciones, delimitaciones y características que poseen cada uno de los empleados.

El Consorcio Metro Bastión es una empresa relativamente joven que tiene ocho años de operaciones, funcionando bajo el sistema integrado de transportación urbana de la ciudad de Guayaquil, es por esto que la gestión de los procesos administrativos como los que tienen que ver con el departamento de talento humano se los realiza de forma empírica e informal, y claramente se evidencia la carencia de una directriz que le permita orientar el esfuerzo diario de todo su personal en resultados favorables a toda la organización.

Está ubicada en el cantón Guayaquil provincia del Guayas en el Kilómetro 11,5 de la Vía Daule y la Avenida Marcel Laneado de Wind, diagonal a la ciudadela Montebello, la empresa es dirigida por una junta de accionistas, un apoderado general, un gerente general, cuenta con seis departamentos con un jefe respectivamente, el total de trabajadores es de 450 personas entre administrativos y operativos distribuidos de la siguiente manera.

- Departamento de Operaciones, 345 trabajadores.

- Departamento de Recaudaciones, 23 trabajadores.

-Departamento de Mantenimiento, 71 trabajadores.

- Departamento de Talento Humano, 6 trabajadores.

-Departamento de Bodega, 4 trabajadores.

- Departamento de Seguridad y Salud Ocupacional, 1 trabajador. 
Actualmente la empresa no cuenta con estudios o información similar al presente tema de investigación que permitan dar alguna referencia de la misma, por lo tanto, como antecedente se cuenta con datos referente a la empresa proporcionados por el personal con más antigüedad en los puestos de trabajo. El principal objetivo de esta investigación es hacer un estudio minucioso y analizar las falencias en las que incurre el departamento de talento humano, como son la ejecución de sus procesos administrativos y elaborar un modelo que permita desarrollar conocimientos, habilidades y técnicas en los puestos de trabajo, esto contribuirá para definir elementos y procedimientos claves para el diseño de gestión administrativo de talento humano, lo que es importante para toda empresa ya que mejora su productividad.

El fundamento teórico obedece a direccionar los esfuerzos que se requieren con la finalidad de ofrecer un aporte teórico basado en una metodología coherente a las necesidades de la empresa.

Esta investigación es realizada con la finalidad de proponer un diseño administrativo para el departamento de talento humano, ya que no cuenta con herramientas administrativas que le permitan un mejor desarrollo en sus procesos, basado en directrices que permitirán dar un mayor conocimiento y realce a sus procedimientos, obteniendo personal calificado y apto para desempeñarse en el puesto de trabajo creando eficiencia y una cultura emprendedora.

Uno de los problemas que se observa en el departamento de talento humano es el desorden en la ejecución de los procesos de selección y contratación de personal, la falta de un efectivo plan de desarrollo y capacitación, la informalidad en el proceso de desvinculación y la falta de registros; sumado a esto la ausencia de medición de efectividad en estos procesos.

En la actualidad los cambios en el entorno empresarial han generado nuevos modelos administrativos enfocados a obtener una ventaja competitiva basado no solo en la tecnología y en la optimización de sus procesos, sino en los valores organizacionales que recaen en el capital, con este trabajo investigativo se logrará dirigir de forma eficiente los procesos del departamento de talento humano que ad- ministre en función de las necesidades y objetivos estratégicos que demande la organización, ofreciendo aplicación práctica para que la empresa pueda diseñar una herramienta altamente efectiva para la gestión del talento humano y para ello se establece una metodología a seguir.

\section{METODOLOGÍA}

La metodología se estableció de la siguiente manera:

- Como primer paso se realiza el diagnostico situacional de la empresa, para mostrar cual es el escenario actual, y con esto identificar como es la gestión del talento humano.

- Se realiza una planificación para el desarrollo de la gestión del talento humano.

- Se hace el desarrollo de la guía para el diseño de los procesos de la gestión de talento humano del consorcio Metro Bastión.

-Formulación de la misión, visión y valores, con las que no cuenta el departamento de talento humano.

-Se establecen las conclusiones.

Esta investigación hace un análisis descriptivo y estadístico permitiendo contrastar e identificar el comportamiento de datos cuantitativos a través de los resultados obtenidos del instrumento científico (encuesta) dirigida al personal administrativo y operativo de la empresa Consorcio Metro Bastión, teniendo como objetivo tabular los datos de las encuestas, que fueron empleadas a 208 colaboradores entre personal administrativo y operativos, quienes proporcionaron la información, que sirve para el análisis e interpretación de los datos y realizar las conclusiones respectivas.

En la empresa laboran 450 personas, tomándose una muestra exacta a encuestar, que ayudaran a verificar la situación actual de la empresa.

Para obtener los resultados de muestra de Población Finita se aplica la siguiente forma y sus respectivos datos: 
$\mathrm{N}=$ Total de la población 450 personas

$\mathrm{Z}^{2} \mathrm{u}=1.96$ al cuadrado (si la seguridad es 95\%)

$\mathrm{d}^{2}=$ Grado de error $5 \%$

$(p)=$ Nivel de ocurrencia $50 \%$

$(q)=$ Nivel de no ocurrencia $1-p$

$\mathrm{n}=$ Tamaño de la muestra $\dot{ }$ ?

Con los siguientes datos se procede a aplicar la siguiente formula.

$$
\mathrm{n}=\frac{\mathrm{N}^{*} \mathrm{Z}_{\mathrm{u}}^{2} \mathrm{p}^{*} \mathrm{q}}{\mathrm{d}^{2} *(\mathrm{~N}-1)+\mathrm{Z}_{\mathrm{u}}^{2} \mathrm{p}^{*} \mathrm{q}}
$$

Donde:

$\mathrm{N}=$ Tamaño de la muestra $i ?$

$Z^{2} \mathrm{u}=$ Nivel de confianza $95 \%=1.96^{2}$

$\mathrm{d}^{2}=$ Presión (de la investigación $\left.5 \%=0.05\right)$

$\mathrm{p}=$ Variable positiva (en este caso se utilizó el $50 \%=0.5$ )

$\mathrm{q}=$ Variable negativa $50 \%=0.5$

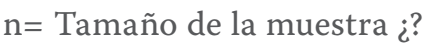

$n=\frac{(450)(0,5)^{2}(1,96)^{2}}{\left[(450-1)(0,05)^{2}\right]+\left[(0,5)^{2}(1,96)^{2}\right]}=$

\section{$\mathrm{n}=\frac{432.18}{2,0829}=\mathbf{2 0 8}$}

El estudio se encuentra dentro del paradigma cuantitativo como indican (Huber, Fernández, \& Lorenzo, 2001), los programas de análisis de datos cualitativos únicamente sirven para lograr sistematizar y controlar el proceso de análisis de datos, por lo que corresponde al investigador asignar los significados oportunos a esos datos.

Se usa el método cuantitativo, al respecto (Mendoza, 2006) hace referencia a que los métodos cuantitativos surgen en los siglos XVIII y XIX, como elementos dentro del capitalismo, para analizar los conflictos sociales y económicos como un todo complejo, además hace mención de que la inves- tigación cualitativa está inspirada en las ciencias naturales y a su vez, en la física new-toniana.

\section{RESULTADOS}

Según la encuesta realizada al personal: ¿cree usted que existen mecanismos formales para la revisión, mejoramiento y actualización de los procedimientos de la empresa?, el $54,33 \%$ de las personas que fueron encuestadas responden que no existen mecanismos formales para el mejoramiento, y el 45,67\% indican que, si hay mejoramiento en los procesos y procedimientos de la empresa, por lo tanto, se debería mejorar de manera continua su desempeño laboral y calidad.

El 37\% de las personas indican que, si se realizan los procesos administrativos adecuados, y en mayor proporción las personas han manifestado que el 62,50\% afirman que la gestión de procesos con el que cuenta la empresa no es eficiente para realizar una correcta administración de su Talento Humano.

Se pudo determinar que el 41,83\% si está de acuerdo con la forma de selección de personal según su capacidad técnica y profesional para desempeñar un cargo, mientras que el 58,17\% se encuentra en desacuerdo ya que se lleva el proceso de selección de una manera informal y no se cumple con la capacidad técnica y profesional del personal que labora en la empresa

De los análisis arrojados por los resultados de las encuestas realizadas al personal que labora en las diferentes áreas de la empresa consorcio Metro Bastión de Guayaquil, se concluye que los inconvenientes y falencias con las que cuenta el departamento de talento humano, repercute en el desarrollo del personal que labora en la empresa

En la empresa consorcio Metro Bastión de Guayaquil se tiene desconocimiento de los procesos, procedimientos, misión, visión y la aplicación de manuales y guías para cada departamento, necesariamente tienen que ser bien estructurados y detallados donde se indiquen y definan las funciones necesarias dentro de la estructura de la organización. que garanticen todas las actividades y tareas que deben ejecutar el personal de la empresa, para el cumplimiento de su objeto social y su misión, de igual manera estos procesos deben 
ser difundidos al personal y los funcionarios de la organización.

Con la presente investigación se pudo constatar la necesidad de tener procesos administrativos bien estructurados para el departamento de talento humano, y de esta manera contar con un personal calificado que colabore eficientemente a la productividad de la empresa.

Para (Mondy, 2005) el desarrollo de los recursos humanos -DRH- representa una función de suma importancia, consistente en la capacitación, actividades de planeación y desarrollo de carreras individuales, desarrollo organización y evaluación del desempeño.

Para alcanzar resultados plenamente satisfactorios en la aplicación de estos conceptos se requiere, como recomienda la Dra. Julia Añorga: “...identificar las competencias que el mercado laboral requiere de los profesionales y en base a ello diseñar programas de estudio adecuados que contemplen los requerimientos que demanda la sociedad de hoy" (Añorga Morales, 2012) y recomienda, que esta identificación se realice “...mediante las indagaciones empíricas, [de manera que] podamos determinar cuáles y como deben ser las nuevas competencias que se proponen..." (Añorga Morales, 2012) criterio que a juicio del autor alude al papel de los centros de capacitación en su labor investigativa y científica, vinculados directamente a los centros de producción o servicios.

Según lo que indica el análisis de las encuestas, la falta de capacitación al personal es muy visible, por lo que se debe tomar en consideración los diferentes aspectos, ya que la mayor parte del personal requiere capacitación de acuerdo a su actividad laboral, estas capacitaciones deben responder a las necesidades de cada trabajador de forma planificada y constante que permita la actualización de la empresa de forma especializada, que obtenga el conocimiento requerido y puedan ser más eficientes en sus áreas de trabajo.

En consecuencia, (Porter, 2005) afirma que la competitividad se define por la productividad con la cual un país utiliza sus recursos humanos, económicos y naturales, coincidiendo con la propuesta de (Mercado, 1998) quien añade que la productividad en términos empresariales es la relación entre el nivel de producción y los insumos utilizados para obtenerla, asociada a la eficiencia en el manejo de los recursos, es decir, al mejoramiento continuo de los métodos de producción y la adaptabilidad al cambio permanente de las situaciones.

De acuerdo con los planteamientos de enfoque Poder-Seeo, propuesto por (Martínez, 2005) la investigación debe servir para evaluar y retroalimentar los resultados para dar solución a los problemas, señalando la importancia que tienen las variables para los gerentes, para mejorar el desempeño de sus empresas, las cuales se basan en funciones de relaciones verticales y transversales (de acuerdo a la estructura empresarial) orientadas a la planeación según los objetivos de calidad de la producción, eficacia social y sostenibilidad, procesos de organización orientados a la auto estructuración, direccionamiento del desarrollo del potencial humano, y procesos de realimentación en interacción con la estructura externa que orientan las actividades de investigación, aprendizaje e innovación de conocimiento en interacción con una estructura de organizaciones de negocios, en busca siempre de una ventaja competitiva.

Por otra parte, se debe informar a los directivos de la empresa, dándoles a conocer que no se cuenta con herramientas administrativas y tecnológicas en cuanto a sistemas operativos de los departamentos, que permita el mejor desempeño laboral en la empresa con esto se reduciría pérdida de tiempo permitiendo trabajar de una manera más eficiente y productiva.

Por lo anteriormente expuesto es preciso agrupar esfuerzos desde diferentes instituciones para capacitar a dueños, gerentes y personal de la empresa y mostrar casos triunfantes, divulgar resultados de investigaciones relacionadas, con el fin de sensibilizar y mostrar que sí es posible lograr mejoras significativas. De esta manera y ligados a las tendencias empresariales, una entidad que no sea capaz de ajustarse a los cambios del entorno empresarial, de precisar estrategias basadas en un enfoque de gestión integral y de dirección, y que no se proyecte, difícilmente será capaz de sostenerse y continuar en el mercado. 
Hoy en día la gestión del talento humano no solo se trata de procesos que servirán para obtener utilidades a los propietarios de la empresa, sino que envuelven una serie de recursos entre ellos uno de los más importantes como lo es el factor humano, son ellos el motor fundamental de toda organización quienes con su fortaleza, conocimiento y toma de decisiones guían y motivan al resto de personal para dar un valor agregado a la organización.

El consorcio Metro Bastión de Guayaquil se ha enfocado en maximizar sus ingresos, y se han despreocupado del bienestar de sus colaboradores, es por ello que existe mucha rotación de personal e inseguridad en el trabajo, se verificó que no cuentan con clima laboral adecuado, y a su vez sus necesidades laborales no son satisfechas.

Las estrategias organizacionales son acciones que se realizan para mantener y soportar el logro de objetivos de la organización, unidad de trabajo, encaminada a conseguir algo deseado por varios competidores, teniendo en cuenta los recursos y capacidades de las cuales se dispone (Serna, 2008)y (Garrido, 2006). Competir de manera diferente; hacer lo que los competidores no hacen o, mejor, hacer lo que no pueden hacer. Las opciones estratégicas de una compañía rara vez son sencillas y a menudo requieren cambios difíciles, pero no es excusa para abstenerse de tomar decisiones de un curso concreto de acción (Collis, 2008).

$\mathrm{Si}$ la gerencia hace referencia al quehacer del rol directivo, relacionado con la conducción de la organización para el desarrollo de su misión en función del logro de la visión (Bernal, 2013), los estilos gerenciales por lo general se asocian con liderazgo concebido como la forma de influir en los demás para lograr las metas propuestas, siendo reflejo de la exigencia al trabajador para alcanzar los objetivos deseados.

De igual forma, se habla de competencias gerenciales, definidas como conjunto de saberes puestos en juego por los directores y gerentes para resolver situaciones concretas de dirección y coordinación en la organización. (Gutiérrez, 2010. )las clasifica en diez grupos resumidas en habilidades de dirección, servicio al cliente, efectividad interpersonal, toma de decisiones, trabajo en equipo, desarrollo de personas, liderazgo, pensamiento estratégico, capacidad de negociación y orientación al logro. Recuerda (Senge, 2000) que, todo sistema empieza desde la pequeñez, el ser humano comienza como un embrión, si se quiere efectuar cambios dentro de una organización, se debe empezar con un grupo piloto, empezar a pequeña escala y crear procesos de auto refuerzos que apoyen, pensar en iniciar, sostener y difundir estrategias de innovación administrativas.

La presente investigación sirve para la búsqueda de personal con alto grado de creatividad, capacidad y talento que aporten a la innovación y cumplimiento de los objetivos trazados por el consorcio Metro Bastión de Guayaquil, es por ello que se implementan nuevas técnicas de conocimiento, capacitación y entrenamiento que les permitan llevar de manera directa la información a cada trabajador y de esta manera mejorar la relación empleador a trabajador.

Con relación a la Cultura (Robbins, 2009) dice que esta, fomenta el compromiso con la organización y la coherencia del comportamiento de los trabajadores se incrementa, y esto beneficia a la empresa.

\section{CONCLUSIONES}

Con la finalización de la investigación se da a conocer la magnitud de la importancia de los elementos que aportan al mejoramiento en el desarrollo de la organización, tales como son la misión, visión tanto de la empresa como de los departamentos, los procesos administrativos, los reglamentos y sus respectivos manuales de funciones, que contribuirán eficientemente al desarrollo de las metas y objetivos establecidos por la empresa.

Claramente se ha podido demostrar que un departamento con falencias en la ejecución de sus procesos desencadena en un inadecuado uso de sus recursos, contrataciones de personal con bajo rendimiento y falta de herramientas administrativas que permitan un buen desarrollo en la evaluación de su personal.

Herramientas técnicas y modernas como manuales de funciones que ayuden al mejor desempeño en la gestión de talento humano, mediante la evaluación de los puestos y así se logrará contratar personal adecuado para el puesto requerido. 
Un efectivo plan de capacitación ayuda a que se realicen inducciones en el proceso de selección del personal, por otra parte, no cuentan con actualizaciones periódicas en los procesos informales que tiene la empresa, el personal no se encuentra motivado ya que las empresas no brindan mecanismos para ascender laboralmente y profesionalmente.
Algunas de las necesidades que más relevancia tuvieron son las condiciones y el ambiente laboral en los puestos de trabajo que no son las adecuadas, además se manifestó que no se toma en consideración al trabajador para una correcta remuneración y muchas veces no se cuentan con los beneficios de ley como horas extras.

\section{REFERENCIAS BIBLIOGRÁFICAS}

Añorga Morales, J. A. (2012). La Educación Avanzada y el Mejoramiento Profesional y Humano. La Habana: UCP. "Enrique J. Varona".

Arias, F. G. (2012, p. 82). El proyecto de Investigacion. Caracas: Episteme C.A.

Baguer, Á. (2001). Un timón en la tormenta. Cómo implantar con sencillez la gestión de los recursos humanos en las empresas. Madrid, España: Ediciones Díaz de Santos, S.A.

Bernal, C. S. ( 2013). Proceso administrativo para las organizaciones del siglo XXI. . Bogotá. Colombia : Pearson.

Collis, D. y. ( 2008). Can You Say WhatYour Strategy Is? . Harvard Business Review 86,núm. 4, pp. 82-90.

Chiavenato, I. (2009). Gestión del talento humano. Colombia: McGraw Hill.

Chiavenato, I. (2009). Administración de Recursos Humanos. (5ta. Edición). Colombia: McGraw Hill.

Fayol, H. (1982). Administración industrial. México: Herrero Hermanos.

Fernández, J. ( 1999.). Liderazgo natural. Cuadernosde management para una dirección eficaz. . DeustoBilbao. España.

Garrido, S. ( 2006). Dirección estratégica. McGrawHill.

Gulick, L. (1992). Notes on the Theory ofOrganization", en GULICK, L. y L. Urwick (eds.),Papers on The Science of Administration, NuevaYork: Institute of Public Administrationcitado por CHIAVENATO, Idalberto, Introducción ala teoría general de la administración. México: McGraw-Hill.

Gutiérrez, E. (2010. ). Competencias gerenciales. Habilidades conocimientos y aptitudes. Bogotá Colombia: Ecoeediciones. .

Huber, G., Fernández, G., \& Lorenzo, O. y. (2001). Análisis de datos cualitativos con Aquad Cinco. Granada: Grupo Editorial Universitario.
Likert, R. ( 1975). El factor humano en la empresa: Su dirección y valoración. . Deusto. Bilbao. España.

Martínez, C. E. ( 2005). Teoría avanzada de organización y gestión. Análisis del desarrollo de competencias en empresas colombianas. . Bogotá. Colombia: Universidad Nacional de Colombia. .

Mendoza, R. (2006). Investigación cualitativa y cuantitativa. Diferencias y limitaciones.

Mercado, E. ( 1998). La productividad base de la competitividad. México.: Limusa. Noriega editores.

Mondy, R. y. (2005). Administración de recursos humanos. Novena Edición. México: Pearson Educacion.

Porter, M. ( 2005). ¿Qué es la competitividad? CentroAnselmo Revírala de Globalización y Estrategia,IESE Business School. Barcelona España. : Universidad de Navarra.

Quintanilla, I. (2002). Empresas y personas: Gestión del conocimiento y capital humano. España: Ediciones Díaz de Santos.

Robbins, S. y. (2009). Comportamiento Organizacional. México: Pearson Educacion.

Rodríguez, V. J. (2007). Administración Moderna de personal.(7a. Edición). México: CengageLearning Editores, S.A.

Senge, P. (2000). La danza del Cambio: Los retos de sostener el impulso en organizaciones abiertas al aprendizaje. Colombia: Editorial Norma, S.A.

Serna, H. ( 2008). Gerencia estratégica. . Bogotá, Colombia.: Panamericana editorial Ltda.

Thompson, A., M, P., \& Gamble, J. y. (2012). Administración estratégica. . México. D.F.: McGrawHill18 a edición. 